\title{
Aerosol Science and Technology
}

\section{Comparing Aerosol Concentrations and Particle Size Distributions Generated by Singing, Speaking and Breathing}

Florence K. A. Gregson, Natalie A. Watson, Christopher M. Orton, Allen E. Haddrell , Lauren P. McCarthy, Thomas J. R. Finnie, Nick Gent, Gavin C. Donaldson, Pallav L. Shah, James D. Calder, Bryan R. Bzdek, Declan Costello \& Jonathan P. Reid

To cite this article: Florence K. A. Gregson, Natalie A. Watson, Christopher M. Orton, Allen E. Haddrell , Lauren P. McCarthy, Thomas J. R. Finnie, Nick Gent, Gavin C. Donaldson, Pallav L. Shah , James D. Calder , Bryan R. Bzdek, Declan Costello \& Jonathan P. Reid (2021): Comparing Aerosol Concentrations and Particle Size Distributions Generated by Singing, Speaking and Breathing, Aerosol Science and Technology

To link to this article: https://doi.org/10.1080/02786826.2021.1883544

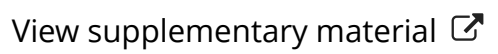

Accepted author version posted online: 05

Feb 2021.

Submit your article to this journal

Q View related articles $\sqsubset$

View Crossmark data $\asymp$ 


\section{Comparing Aerosol Concentrations and Particle Size Distributions Generated by Singing, Speaking and Breathing}

Florence K. A. Gregson, ${ }^{1}$ Natalie A. Watson, ${ }^{2}$ Christopher M. Orton, ${ }^{3,4,5}$ Allen E. Haddrell, ${ }^{1}$ Lauren P. McCarthy, ${ }^{1}$ Thomas J. R. Finnie, ${ }^{6}$ Nick Gent, ${ }^{6}$ Gavin. C. Donaldson, ${ }^{5}$ Pallav L. Shah, ${ }^{3,4,5}$ James D. Calder, ${ }^{7,8}$ Bryan R. Bzdek, ${ }^{1}$ Declan Costello ${ }^{9,}$ and Jonathan P. Reid ${ }^{1,}$

${ }^{1}$ School of Chemistry, University of Bristol, Bristol, United Kingdom

${ }^{2}$ Department of Ear, Nose and Throat Surgery, Lewisham and Greenwich NHS Trust, London, United Kingdom

${ }^{3}$ Department of Respiratory Medicine, Royal Brompton Hospital, London, United Kingdom

${ }^{4}$ Department of Respiratory Medicine, Chelsea \& Westminster Hospital, London, United Kingdom

${ }^{5}$ National Heart and Lung Institute, Guy Scadding Building, Imperial College London, London, United Kingdom

${ }^{6}$ Emergency Response Department Science and Technology (ERD S\&T), Health Protection Directorate, Public Health England, Porton Down, Salisbury, Wiltshire, United Kingdom

${ }^{7}$ Department of Bioengineering, Imperial College London, United Kingdom

${ }^{8}$ Fortius Clinic, London, UK

${ }^{9}$ Ear, nose and throat department, Wexham Park Hospital, United Kingdom

CONTACT Declan Costello d.costello@nhs.net Ear, nose and throat department, Wexham Park Hospital, SL2 4HL, UK;

Jonathan P. Reid j.p.reid@bristol.ac.uk School of Chemistry, University of Bristol, Bristol, BS8 1TS, UK.

\section{ABSTRACT}

The severe acute respiratory syndrome coronavirus 2 (SARS-CoV-2) pandemic has resulted in an unprecedented shutdown in social and economic activity, with the cultural sector particularly severely affected. Restrictions on musical performances have arisen from a perception that there is a significantly higher risk of aerosol production from singing than speaking, based upon high-profile 
examples of clusters of COVID-19 following choral rehearsals. However, comparing aerosol generation from different types of vocalization, including singing, across a range of volumes is a rapidly evolving area of research. Here, we measured aerosols from singing, speaking and breathing from a large cohort of 25 professional singers in a range of musical genres in a zero-background environment, allowing unequivocal attribution of aerosol production to specific vocalizations. We do not assess the relative volumes at which people speak and sing. However, both showed steep increases in mass concentration with increase in loudness (spanning a factor of 20-30 across the dynamic range measured, $p<0.001)$. At the quietest volume (50 to $60 \mathrm{dBA})$, neither singing $(p=0.19)$ nor speaking $(p=0.20)$ were significantly different to breathing. At the loudest volume (90 to $100 \mathrm{dBA})$, a statistically significant difference $(p<0.001)$ was observed between singing and speaking, but with singing only generating a factor of between 1.5 and 3.4 more aerosol mass. Guidelines for musical performances should be based on the loudness and duration of the vocalization, the number of participants and the environment in which the activity occurs, rather than the type of vocalization. Mitigations such as the use of amplification and increased attention to ventilation should be employed where practicable.

\section{INTRODUCTION}

A novel strain of a human coronavirus was first identified in late 2019, designated severe acute respiratory syndrome coronavirus 2 (SARS-CoV-2), and is responsible for the global outbreak termed coronavirus disease 19 (COVID-19) (Huang et al. 2020; Wu and McGoogan 2020; Wu et al. 2020). Pandemic status was declared on 11 March 2020 by the World Health Organization (WHO), with in excess of 51.8 million cases and 1.28 million deaths reported worldwide by $13^{\text {th }}$ November 2020 (World Health Organisation 2020). Early in the pandemic, clusters of COVID-19 were considered to have arisen in several choirs around the world (Charlotte 2020; Miller et al. 2020). This rapidly led to many governments restricting or suspending singing. Concerns that woodwind and brass instruments might also be responsible for virus spread led to similar restrictions on the playing of wind instruments. Consequently, large sections of the cultural sector, along with religious institutions and educational establishments, were unable to rehearse and perform, resulting in profound artistic, cultural, spiritual, emotional and social impacts. The livelihoods of many performers have been jeopardized, and the viability of established institutions remains threatened. The economic impact to the United Kingdom (UK) from this sector alone has been substantial, costing the UK economy hundreds of millions in lost 
tax revenue, usually derived from the $£ 32.2$ billion cultural purse (Department for Digital Culture Media \& Sport 2018).

Respiratory particulate matter is expelled during human exhalatory events, including breathing, speaking, coughing and sneezing (Asadi et al. 2019; Johnson et al. 2011; Morawska et al. 2009). The flux generated is proportional to the amplitude of phonation in speech (Alsved et al. 2020; Asadi et al. 2020). These actions release a plume of material containing particles of varying size, ranging from macroscopic mucosalivary droplets originating from the oral cavity and pharynx, to microscopic aerosols released by the small airways of the lungs (Asadi et al. 2020; Johnson et al. 2011; Morawska et al. 2009; Papineni and Rosenthal 1997). Traditionally, the division between droplets, which are considered to be of sufficient mass to sediment due to gravity, and aerosols, which remain airborne, is defined arbitrarily at $5 \mu \mathrm{m}$ diameter (Xie et al. 2007; Nicas et al. 2005). However, particle composition and environmental properties like temperature, humidity and airflow influence the biophysical mechanics of the material released and the extent of transport (Tellier et al. 2019; Zayas et al. 2012; Xie et al. 2007; Nicas et al. 2005).

Droplets and airway secretions are established vectors of SARS-CoV-2, with expelled infectious material either directly inhaled by an individual in close proximity, or indirectly transmitted through contact with settled-out fomites (Pan et al. 2020; Woelfel et al. 2020). The role of airborne transmission by respirable aerosol particles is gaining prominence (Morawska and Cao 2020). Viral RNA has been detected in airborne samples collected both inside and outside the rooms of COVID-19 patients (Chia et al. 2020; Guo et al. 2020; Lednicky et al. 2020; Santarpia et al. 2020), and SARS-CoV-2 RNA has been reported in size-resolved aerosol distributions in two hospitals in Wuhan, China (Liu et al. 2020). Retrospective studies of COVID-19 clusters, including a shopping mall, a restaurant and a high-profile outbreak in an American choir group, found no direct or indirect interaction among the individuals contracting the virus, suggesting airborne transmission (Cai et al. 2020; Lu et al. 2020; Miller et al. 2020). SARS-CoV-2 and other viruses, including severe acute respiratory syndrome coronavirus (SARS-CoV-1) and Middle East Respiratory Syndrome coronavirus (MERS-CoV), are stable in aerosol (Schuit et al. 2020; Smither et al. 2020; van Doremalen et al. 2020). Infective airborne potential from human exhalation has been confirmed in other viruses, including respiratory syncytial virus, influenza and MERS-CoV (Yan et al. 2018; Lindsley et al. 2016). 
Several online reports have attempted to examine the quantities of particulate matter expelled by participants performing a range of activities including singing but have struggled to accurately quantify aerosol and droplets because of the large number of background particulates in the environment. This is the largest study to date with the objective of comparing the relative amounts of aerosols (up to $\sim 10 \mu \mathrm{m}$ diameter) generated by singing and speaking at the same loudness, and the first to present results from different genres, complementary to other studies with similar experimental aims (Alsved et al. 2020). We present results from a large cohort of 25 professional performers completing a range of exercises including breathing, speaking, coughing and singing in the clean air environment of an operating theatre with laminar flow ventilation. Measurements of particle number concentration alone would be insufficient to determine the total amount of viral material capable of being transmitted: the total mass of particulate matter produced may be a key factor in assessing the potential risk. Thus, measurements of particle size distributions, as well as concentration, are used to assess the mass concentration.

\section{MATERIALS AND METHODS}

\section{Experimental Design}

Aerosol concentrations were measured from singing, speaking and breathing in a zero-background environment. The cohort of 25 professional singers performed a broad range of genres, including musical theatre (6), choral (5), opera (5), and other genres: gospel (2), rock (2), jazz (2), pop (1), actor with singing interest (1) and soul (1). 6 identified their voice-type as soprano or mezzo-soprano, 7 as alto, 5 as tenor and 7 as bass or baritone. Aerosol and droplet concentrations were measured with an Aerodynamic Particle Sizer (APS, $500 \mathrm{~nm}-20 \mu \mathrm{m}$, see Aerosol Measurements) in an operating theatre with each participant and researcher required to wear appropriate personal protective equipment (hospital scrubs, theatre hat, and face mask if not the singing participant) (Figure 1a). The operating theatre had an ultra-clean ventilation system (e.g. EXFLOW 32; Howorth Air Technology, Farnworth, UK) which generates a surgical canopy of filtered air vertically downwards. Typically, the filter runs at $50 \mathrm{~Hz}$, leading to a high air exchange rate of 500 - 600 air changes per hour. This system reduced the pre-existing particle background number concentration to zero $\mathrm{cm}^{-}{ }^{3}$. Thus, any particles detected were directly attributable to participant activity, with particle concentrations returning to zero $\mathrm{cm}^{-3}$ between periods of singing, speaking and breathing (Figure 1b). Temperature and relative humidity were typically $20^{\circ} \mathrm{C}$ and $45 \%$, respectively. 
A standard operating procedure was adopted (see Vocalization experiments) covering 12 activities over $\sim 1$ hour, with each activity involving up to 5 repeat actions, with a 30 s pause between each repetition. These activities included breathing, coughing, singing single notes (“/a/") at different pitches, and speaking and singing the "Happy Birthday" song at different loudness. At the beginning of each action, participants stepped forward to the funnel (Figure 1a) such that the dorsum of the nose was aligned to the plane of the base of the cone. Participant position relative to the funnel was monitored to ensure consistency (within $10 \mathrm{~cm}$ of the sampling tubes) across all measurements (Figure S1). As in previous studies (Alsved et al. 2020; Asadi et al. 2019; Johnson et al. 2011), we report concentrations sampled through the collection funnel, which allows comparison of particle emission rates on a relative basis between activities. In reality, particle concentrations will become rapidly diluted once particles are exhaled, leading to strong spatial variations.

[Figure 1]

\section{Human Subjects}

The Public Health England Research Ethics and Governance of Public Health Practice Group (PHE REGG) approved this study, and all research was performed in accordance with relevant guidelines and regulations of the Ethical Review Board. We recruited 25 healthy volunteers (12 males and 13 females), ranging in age from 22 to 57 years old (mean 38, SD +/- 9.8) through contact and collaboration with the entertainment industry. Informed consent was obtained from all participants prior to study participation. All participants completed a pre-screening questionnaire including age, gender, professional status, singing training history and COVID-19 symptom status, to fulfill inclusion/exclusion criteria. Only participants who self-reported no symptoms of COVID-19 and who had normal temperatures on the day of attendance were included.

\section{Aerosol Measurements}

Measurements were performed simultaneously with two APS instruments (APS 3321 from TSI Incorporated, Minnesota, USA. Sampled at 1 L.min-1 with sheath flow 4 L.min-1, Particle diameters $500 \mathrm{~nm}-20 \mu \mathrm{m}$ ) sampling from the same custom-printed funnel. A comparison of measurements 
between the two APS instruments was linear, with a slope that deviated from 1 owing to different sensitivities of the instruments (Figure S4). Only aerosol concentrations sampled from one APS, the more sensitive of the two, are reported in this paper. The loudness of vocalization was recorded in dBA using a decibel meter (UNI-T, UT353), positioned $\sim 30-40 \mathrm{~cm}$ in front of the participant at eye level.

The sampling funnel was 3D printed from Polylactic acid (1.75 mm filament) by a RAISE3D Pro2 Printer (3DGBIRE). The funnel was $150 \mathrm{~mm}$ wide, $90 \mathrm{~mm}$ deep with ports at the neck for sampling aerosol into the two APS instruments. All tubing was conductive silicone and $130 \mathrm{~cm}$ in length (TSI Inc., product number 3001788 , inner diameter 0.19 inch, outer diameter 0.375 inch).

Previous studies attempted to minimize the sampling distance between the participant and the APS detection region (Asadi et al. 2019); logistical constraints required that we instead used conductive tubing to sample the aerosol from the performer into the APS. Not only did this allow space for the subject to stand comfortably next to the aerosol instrumentation in a normal performance posture, but it facilitated rapid set-up and dismantling of the instrumentation, essential within the frequently used operating theatre without the need for specialist platforms to mount the instruments. In addition, aerosol concentrations were measured in the same studies from brass and woodwind instruments; using flexible tubing facilitated the use of the same experimental set-up for both singing and for musical instrument, thus allowing direct comparison of aerosol concentrations between singer and instrumentalist. The conductive tubing was intentionally positioned to avoid tight curvature in the bends of the tube, maintaining a ratio of the radius of curvature $\left(r_{c}\right)$ to the inner tube radius $\left(r_{t}\right)$ greater than 50. Work by Cheng and Wang has reported that the impaction loss of aerosol number concentration passing around tube corners for $r_{c} / r_{t}>30$ is less than $13 \%$ for the particles of diameter $<7 \mu \mathrm{m}$ and less than $30 \%$ for the particles of diameter $<11 \mu \mathrm{m}$ when sampled at the Stokes numbers and flow rates characteristic of the APS (Stokes numbers $<0.14$ and $<0.36$ for the lower and upper sizes, respectively) (Cheng and Wang 1981). We have measured the transmission efficiency of aerosol sampled through the funnel and conductive tubing and these data are reported and discussed in the Supplemental Material (Figure S5). We did not make additional corrections to the sampled aerosol concentrations based on this transmission efficiency at different particle sizes.

\section{Vocalization Experiments}




\section{"/a/" experiments}

Participants voiced /a/ (the vowel sound in 'far') for $10 \mathrm{~s}$ at 70-80 dBA in close proximity to the funnel followed by $30 \mathrm{~s}$ of nose breathing and standing $2 \mathrm{~m}$ away from the funnel, repeated four more times in succession. The participant repeated the series of five / $a /$ vocalizations at the same amplitude using feedback from a decibel meter. Soprano/mezzo soprano singers sang note F4, alto note D4, tenor note F3 and baritone/bass note C3. After each set of experiments participants were asked to take a sip of water.

This set of experiments was repeated an octave above at 70-80 dBA. Soprano/mezzo soprano singers sang note F5, alto note D5, tenor note F4 and baritone/bass note C4. Timed prompts with directions for the requested vocalization were delivered by the researcher and immediate contemporary guidance given if the amplitude was out of range.

\section{“Happy Birthday” speaking experiments}

Participants spoke the words of the "Happy Birthday" song to "Dear Susan" for $20 \mathrm{~s}$ at 50-60 dBA followed by 30 seconds of nose breathing and standing $2 \mathrm{~m}$ away from the funnel, repeated four more times in succession. The participants then repeated this sequence at 70-80 dBA and at 90-100 dBA.

\section{“Happy Birthday" singing experiments}

Participants sang the "Happy Birthday" song to "Dear Susan" for $20 \mathrm{~s}$ at 50-60 dBA followed by $30 \mathrm{~s}$ of nose breathing and standing $2 \mathrm{~m}$ away from the funnel, repeated four more times in succession. The participants then repeated this sequence at 70-80 dBA and at 90-100 dBA. Soprano/mezzo soprano singers sang in B flat major (starting note F4, top note F5), alto in G major (starting note D4, top note D5), tenor in B flat major (starting note F3, top note F4) and baritone/bass in F major (starting note C3, top note $\mathrm{C} 4)$.

\section{Breathing experiments}

Participants breathed for $10 \mathrm{~s}$ inhaling through the nose and exhaling through an open mouth in a nonforced "quiet" fashion, then stood $2 \mathrm{~m}$ away from the funnel for $30 \mathrm{~s}$ in between each breathing experiment and repeated four more times. An additional set of five breathing measurements were conducted in similar fashion but where the participants inhaled through the nose and exhaled out of the nose in a "quiet" fashion. 


\section{Confirmatory "/a/" experiments.}

Participants voiced /a/ (the vowel sound in 'far') for ten seconds at 70-80 dBA followed by $30 \mathrm{~s}$ of nose breathing and standing away $2 \mathrm{~m}$ away from the funnel, repeated four more times in succession. The participant repeated the series of five / $\mathrm{a}$ / vocalizations at the same amplitude using feedback from a decibel meter. Soprano/mezzo soprano singers sang note F4, alto note D4, tenor note F3 and baritone/bass note $\mathrm{C} 3$.

\section{Coughing}

Participants were asked to cough into the funnel once, stand $2 \mathrm{~m}$ away for 30 seconds and then repeat this process two more times.

\section{Data and Statistical Analysis}

Data analysis was performed by collating raw data of aerosol counts from the APS instruments in Microsoft Excel and Origin to analyze temporal trends in aerosol concentration, mass concentrations and size distributions. A software custom-written in LabVIEW was used to automate this process for improved efficiency of the data analysis. Measured total particle number concentrations were summed over the period of activity and divided by the duration of the activity, reporting a mean concentration $\left(\mathrm{cm}^{-3}\right)$ with a standard deviation, i.e. the average concentration of particles sampled within the funnel volume during the activity. With coughs requiring $<1 \mathrm{~s}$, no averaging across a time-dependent concentration is possible and only the integrated number concentrations per single cough are reported. Further, particle size distributions were recorded by the APS at $1 \mathrm{~s}$ intervals, with 51 size bins equally

spaced in the range 0.523 to $20 \mu \mathrm{m}$ in $\log$ (diameter) space. Average size distributions were calculated first by determining the mean size distribution for each participant and then calculating the mean and standard deviation across all participant size distributions for each activity. Mass concentrations were calculated assuming particle density was $1000 \mathrm{~kg} \cdot \mathrm{m}^{-3}$. Our reported number concentrations and particle size distributions for speaking and breathing are consistent with previously published data (Asadi et al. 2019). We would caution the reader to recognize that, at the smallest particle sizes, the APS reported concentration is likely to be less accurate than that at the larger sizes. In particular, all particles smaller than $0.523 \mu \mathrm{m}$ are put in a single size bin, providing no discrimination in size. That size bin (smaller 
than $0.523 \mu \mathrm{m}$ ) was excluded from the analysis in this study. Thus, we report aerosol concentrations from 51 size bins, ranging from 0.523 to $20 \mu \mathrm{m}$ in diameter.

The lme package in R-software was used to fit linear random effect models with log-base-e transformed particle concentration or mass as the dependent variable. The independent variables were activity (where loudness was considered as part of activity) or vocalization (speaking or singing) and acoustic loudness (50-60, 70-80 and 90-100 dBA); the random effect was participant identification number in both cases. In the second analysis, an interaction term between vocalization and loudness was considered but not found to be significant, so the final model does not include such a term. In the Figures, the lower and upper hinges (ends of boxes) correspond to the first and third quartile (the 25th and 75th percentiles). The upper whisker extends from the upper hinge to the largest value but no further than $1.5 \times \mathrm{IQR}$ (where IQR is the inter-quartile range, the distance between the $1^{\text {st }}$ and $3^{\text {rd }}$ quartiles). The lower whisker extends from the lower hinge to the smallest value at most 1.5×IQR. Data beyond the ends of the whiskers are "outlying points" and indicated in red. All components of the box plots were calculated based on the logarithmically-transformed data, owing to lognormal nature of the data, but the plotted and tabular values reported are converted back to linear space for clarity.

\section{RESULTS AND DISCUSSION}

\section{Aerosol Number Concentrations from Singing Compared with Other Expiratory Activities}

A complete analysis of the time-averaged total particle number and mass concentrations for all 25 participants is reported in Figure 2 and the absolute results summarized in Table 1; data normalized to the aerosol concentration from speaking at 70-80 dBA are compared in Figure S2 and Table S1. The aerosol number concentration generated across all participants took a log-normal distribution, consistent with the data presented in a previous publication; concentrations must always be positivevalued and a small number of individuals generate a significantly larger aerosol flux than the median (Asadi et al. 2019). This is particularly apparent for breathing, where measurements from individuals spanned almost three orders of magnitude. Indeed, 4 participants produced more aerosol in number concentration while breathing than while speaking at 90-100 dBA. The reproducibility of concentration from singing a single note (70-80 dBA) was not only apparent in single participant data (Figure 1b), but also across the cohort, with median concentrations in good agreement $\left(0.83\right.$ and $0.91 \mathrm{~cm}^{-3}$ at beginning 
and end, respectively). Using a mixed effects model where loudness was implicit (i.e. was considered as part of the activity), at the lowest volume (50-60 dBA), neither singing $(p=0.19)$ nor speaking $(p=0.20)$ was significantly different in particle production to breathing, with median number concentrations of 0.10 (IQR $0.07-0.17$ ), 0.19 (IQR $0.09-0.30)$ and 0.28 (IQR $0.07-0.64) \mathrm{cm}^{-3}$ for speaking, singing and breathing, respectively. Where loudness is explicit and separate from activity a mixed effects model showed a statistically significant $\left(p<1 \times 10^{-5}\right)$ enhanced aerosol number concentration for singing when compared to speaking. This enhancement was small relative to the much larger changes associated with increase in loudness $(p<0.001)$. Aerosol number concentration increased by a factor of 10-13 as loudness increased from 50-60 dBA to 90-100 dBA, suggesting that shouting should be associated with little difference in risk to singing at loud volume. This result is comparable with that from previous works. For example, Asadi et al. who also observed a linear increase in the mean generated aerosol number concentration from speech with increasing sound pressure level (dBA) when reported in log space, as in Figure 2a. They reported a factor of $\sim 6.4$ increase in particle number concentration generated by reading a passage of text at a 'near yelling' volume (95 dBA) compared to the lowest sound pressure they studied (60 dBA) (Asadi et al. 2019). Alsved et al. also observed an increase in aerosol production with increasing sound pressure for two types of vocalization at two volumes. Going from 'normal' to 'high' sound pressure led to a greater sampled aerosol flux for speech (270 to 570 particles.s $^{-1}$, respectively) as well as for singing (690 to 980 particles.s $^{-1}$ ) (Alsved et al. 2020).

[Figure 2]

[Table 1]

The aerosol number concentrations from speaking and singing at 90-100 dBA are compared in Figure 3 for male and female participants and for the different genres with the full cohort. Individual participant comparisons are provided in Figure S3. Using linear mixed effect modelling and separate analyses for gender and genre, there were no significant differences in aerosol production either between genders $(p$ $=0.34$ ) or among different genres ( $p$ (choral different from "other genres") $=0.46, p$ (musical theatre different from "other genres") $=0.25$, and $p$ (opera different from "other genres") $=0.42$ ). The variability among genres (almost a factor of 2 between the lowest and highest median concentrations) may be attributed to the small cohort sizes for each genre, the sensitivity of number concentration to 
loudness and a minority of participants emitting higher concentrations than others (who could be classed as super-emitters) (Asadi et al. 2019).

[Figure 3]

\section{Comparing the Aerosol Particle Size Distributions and Mass Concentrations}

The possibility that singing, speaking and breathing generate aerosol particles of different sizes cannot be inferred by comparing particle number concentrations alone. Instead, we must compare the aerosol size distributions from these activities. Previously, two overlapping modes in the size distribution of particles from speaking and coughing have been identified (Asadi et al. 2019; Johnson et al. 2011). These have been attributed to distinct processes in this expiration process. The mode of lowest size is generated in the lower respiratory tract with a second mode generated in the region of the larynx, expected to be the most important in voicing. Figure 4 reports the variation in mean number concentrations with particle size averaged over the 25 participants and includes the fitted distribution from Johnson et al., reported from a cohort of 15 (Johnson et al. 2011).

Johnson et al. considered the influence of evaporation on the recorded size distribution and the reduction in moisture content that occurs when particles are exhaled from the warm and humid respiratory tract (Asadi et al. 2019). They presented parameterizations for both the uncorrected measured size distribution and also assuming a correction to account for the effect of evaporation on the size distribution (Johnson et al. 2011). In our work, the diameters of the exhaled aerosol droplets can be assumed to have decreased significantly due to evaporation before they were detected by the APS, in much the same way as recognized by Johnson et al. Given the hygroscopic growth curves of respiratory fluids remain uncertain at the very high humidities of the lung, and the $\mathrm{RH}$ and temperature of the airflow entering the APS inlet is ill-defined, we have chosen not to attempt to correct our particle sizes but instead compare our size distributions with the uncorrected parameterization from Johnson et al. in Figure 4a. With an estimated transit time of $\sim 0.2 \mathrm{~s}$ from the sampling volume to the detector, we can assume that most of the size distribution had progressed fully or close to equilibration within this time window (Walker et al. 2020; Haddrell et al. 2019). Indeed, our distribution for speaking is in excellent agreement with the shape of the distribution reported by Johnson et al. for particles larger than $800 \mathrm{~nm}$ diameter up to above $7 \mu \mathrm{m}$ in diameter, and this size distribution is very similar to our observed distribution for singing. Although the absolute concentrations were a factor of $\sim 6$ larger in our 
measurements, it should be recognized that the absolute value carries little meaning, reflecting only the instantaneous value recorded by the APS from the sampling funnel, which will depend on the sampling specifications (Asadi et al. 2019).

[Figure 4]

Measured size distributions for speaking and singing were fitted to bimodal lognormal distributions, consistent with previous studies for speaking, breathing and coughing (Asadi et al. 2019; Johnson et al. 2011; Morawska et al. 2009). The fits for the different levels of loudness and types of vocalization all gave very similar mean diameters and variance for both the lower respiratory tract and laryngeal modes, further supporting the conclusion that speaking and singing can be treated similarly, generating particles of similar size distributions (Table S2). However, both vocalizations generated larger particles than breathing: although the size distribution from breathing was well-represented by a bimodal lognormal distribution, the larger, laryngeal mode has shifted to a smaller diameter and had a narrower variance than for speaking and singing. The mean size distributions were normalized with respect to the greatest value of $\mathrm{d} N / \mathrm{d} \log \left(D_{p}\right)$ for each activity (Figure $4 \mathrm{~b}$ ), demonstrating the increase in concentration of particles generated in the laryngeal mode with respect to those from the lower respiratory tract, as vocalization loudness increased. Figure $4 \mathrm{c}$ compares the size distribution of particles generated from speaking at 90-100 dBA by three individual subjects that spanned the range in aerosol mass generated for this activity. The size distributions highlight the participant-to-participant variation, which spanned more than an order of magnitude in concentration of aerosol generated. However, the general shapes of the bimodal size distributions were similar in all cases with particles detected up to $>10 \mu \mathrm{m}$ diameter, albeit at very low concentration. The similarity in the size distributions ensured that robust comparisons about the relative mass concentrations of aerosols generated by speaking and singing at the same loudness could be made.

The consequences of different size distributions are apparent when aerosol mass concentration is reported (Figure 2b, see Tables 1 and $\mathrm{S} 1$ ). This comparison is most important when considering the potential of the different activities to transmit infection. Speaking and singing generated statistically significant differences in mass concentrations of aerosol at similar level of loudness; however, these were modest (median singing values only a factor of 1.5-3.4 times larger than speaking) relative to the effects of the loudness of vocalization (a factor of 20-30 increase). Converting from a number concentration to a mass concentration for breathing, resulted in the mass concentration range shifting to 
lower values relative to speaking and singing, a consequence of the different size distributions associated with voicing and breathing (median values 24 and 36 times higher for speaking and singing at the highest loudness, respectively, compared with breathing).

\section{CONCLUSIONS}

This study demonstrates that the assessment of risk associated with the spread of SARS-CoV-2 in large groups due to respirable particles from speaking and singing should consider the number and mass concentrations of particles generated by these activities. The statistically significant, yet relatively modest differences detected between the type of vocalization at the loudest volume studied, were eclipsed by the effects of loudness on aerosol production, which varied by more than an order of magnitude from the quietest to loudest volume studied, whether speaking or singing. By contrast, the number of particles produced by breathing covered a wide range (spanning from quiet to loud speaking and singing) but has a size distribution shifted to smaller particle sizes, in principle mitigating some of the potential risk associated with the wider emission range. The results of this study are in agreement with that from Alsved et al., who studied the aerosol concentration generated from a cohort of twelve singers when they individually spoke or sang at two levels of sound pressure (Alsved et al. 2020). Singing was shown to generate a greater particle concentration than speaking at an equivalent sound pressure, but the increase in sound pressure showed a large increase in particle production for both types of vocalization. Our work confirms this conclusion across an increased cohort size that spanned varying musical genres.

We also found that a minority of participants emitted substantially more aerosols than others, sometimes more than an order of magnitude above the median, consistent with the long-tail of a lognormal distribution when viewed in linear-concentration space. This observation is consistent with a previous study (Asadi et al. 2019). However, the highest emitters were not consistently the highest across all activities, suggesting the magnitude of emission from an individual may be highly activity specific. It is unclear why some participants emitted substantially more than others, and further studies are required to better characterize the variability of aerosol emission across the population, as well as the consistency of emission from an individual over time. 
These conclusions have important policy implications in the context of creating guidelines to reduce transmission of SARS-CoV-2. Breathing produces smaller particles than singing and speaking, suggesting that vocalization may carry higher risk than breathing if the potential SARS-CoV-2 dose delivered by an individual infected with the virus scales with particle mass. Size distributions are comparable across speaking and singing at the same loudness and generate relatively similar, yet statistically significantly different, numbers of particles. Most importantly, number concentrations from speaking and singing rise in parallel with increasing loudness. Given that speaking and singing produce numbers of particles of the same order of magnitude, and that increasing loudness increases that number by orders of magnitude, guidelines from public health bodies should focus on the loudness at which the vocalization occurs, the number of potentially infectious participants (source strength), the environment (ventilation) in which the activity occurs and the duration of the rehearsal and period over which performers are vocalizing (Charlotte 2020; Miller et al. 2020). For certain vocal activities and venues, amplification may be a practical solution to reduce the loudness of singing by the performers. Based on the differences observed between vocalization and breathing and given that it is likely that there will be many more audience members than performers, singers may not be responsible for the greatest production of aerosol during a performance, and for indoor events measures to ensure adequate ventilation may be more important than restricting a specific activity.

\section{ACKNOWLEDGEMENTS}

We acknowledge the Working Group for The Investigation of ParticulatE Respiratory Matter to InForm Guidance for the Safe Distancing of PerfOrmeRs in a COVID-19 PandeMic (PERFORM). BRB acknowledges support from the Natural Environment Research Council through grant NE/P018459/1. Dyson, Inc, the Atmospheric Measurement and Observation Facility, and the Health and Safety Executive are acknowledged for APS loans. Fortius Clinic and Shoen Clinic are acknowledged for the provision of the operating theatre space to conduct the measurements. We acknowledge the BBC Singers, Eleven Management and the performers for volunteering to be included in this study. We acknowledge Public Health England and the Department for Digital, Culture, Media and Sport who acted as sponsor and funded the research, respectively.

\section{AUTHOR CONTRIBUTIONS}

FKAG, NAW and CMO are joint first authors on this paper. NAW, CMO, PLS, DC and JPR led in the study design and in securing funding. FKAG, BRB, and JPR collected the data. CMO, NAW and PLS prepared the application and secured ethical approval. NAW, CMO, DC and JC managed the 
registration and coordination of participant volunteers and secured access to the operating theatres. FKAG, LPM, AEH and BRB wrote analysis software and analyzed the data. TF, NG and GCD undertook the statistical analysis. JPR, DC, PLS and JC provided technical guidance and advice. JPR, BRB, NAW, CMO, DC, FKAG and LPM drafted the manuscript. All authors read and approved the final manuscript.

\section{COMPETING INTERESTS}

The authors declare no competing interests.

\section{DATA AVAILABILITY}

Correspondence and requests for materials should be addressed to JPR.

\section{REFERENCES}

Alsved, M., Matamis, A., Bohlin, R., Richter, M., Bengtsson, P.E., Fraenkel,C.J., Medstrand, P., and Löndahl, J. (2020). Exhaled respiratory particles during singing and talking. Aerosol Sci. Technol. 54 (11):1245-1248. doi:10.1080/02786826.2020.1812502.

Asadi, S., Bouvier, N., Wexler, A.S., and Ristenpart, W.D. (2020). The coronavirus pandemic and aerosols: Does COVID-19 transmit via expiratory particles? Aerosol Sci. Technol. doi:10.1080/02786826.2020.1749229.

Asadi, S., Wexler, A.S., Cappa, C.D., Barreda, S., Bouvier, N.M., and Ristenpart, W.D. (2019). Aerosol emission and superemission during human speech increase with voice loudness. Sci. Rep. 9 (1):1-10. doi:10.1038/s41598-019-38808-z.

Cai, J., Sun, W., Huang, J., Gamber, M., Wu, J., and He, G. (2020). Indirect Virus Transmission in Cluster of COVID-19 Cases, Wenzhou, China, 2020. Emerg. Infect. Dis. 26 (6):1343-1345. doi:10.3201/eid2606.200412.

Charlotte, N. (2020). High Rate of SARS-CoV-2 Transmission due to Choir Practice in France at the Beginning of the COVID-19 Pandemic. 1-8. doi:10.1101/2020.07.19.20145326.

Cheng, Y.S. and Wang, C.S. (1981). Motion of particles in bends of circular pipes. Atmos. Environ. 15 (3):301-306. doi:10.1016/0004-6981(81)90032-9.

Chia, P.Y., Coleman, K.K., Tan, Y.K., Ong, S.W.X., Gum, M., Lau, S.K., Lim, X.F., Lim, A.S., Sutjipto, S., Lee, P.H., Son, T.T., Young, B.E., Milton, D.K., Gray, G.C., Schuster, S., Barkham, T., De, P.P., Vasoo, S., Chan, M., Ang, B.S.P., Tan, B.H., Leo, Y.-S., Ng, O.-T., Wong, M.S.Y., Marimuthu, K., and Singapore 2019 Novel Coronavirus Outbreak Research Team (2020). Detection of air and surface contamination by SARS-CoV-2 in hospital rooms of infected patients. 
Nat. Commun. 11 (1):2800. doi:10.1038/s41467-020-16670-2.

Department for Digital Culture Media \& Sport (2018). DCMS Sectors Economic Estimates 2018 (provisional): Gross Value Added.

Guo, Z.-D., Wang, Z.-Y., Zhang, S.-F., Li, X., Li, L., Li, C., Cui, Y., Fu, R.-B., Dong, Y.-Z., Chi, X.Y., Zhang, M.-Y., Liu, K., Cao, C., Liu, B., Zhang, K., Gao, Y.-W., Lu, B., and Chen, W. (2020). Aerosol and Surface Distribution of Severe Acute Respiratory Syndrome Coronavirus 2 in Hospital Wards, Wuhan, China, 2020. Emerg. Infect. Dis. 26 (7). doi:10.3201/eid2607.200885. Haddrell, A., Rovelli, G., Lewis, D., Church, T., and Reid, J.P. (2019). Identifying time-dependent changes in the morphology of an individual aerosol particle from their light scattering patterns. Aerosol Sci. Technol. 53:1334-1351.

Huang, C., Wang, Y., Li, X., Ren, L., Zhao, J., Hu, Y., Zhang, L., Fan, G., Xu, J., Gu, X., Cheng, Z., Yu, T., Xia, J., Wei, Y., Wu, W., Xie, X., Yin, W., Li, H., Liu, M., Xiao, Y., Gao, H., Guo, L., Xie, J., Wang, G., Jiang, R., Gao, Z., Jin, Q., Wang, J., and Cao, B. (2020). Clinical features of patients infected with 2019 novel coronavirus in Wuhan, China. Lancet 395 (10223):497-506. doi:10.1016/S0140-6736(20)30183-5.

Johnson, G.R., Morawska, L., Ristovski, Z.D., Hargreaves, M., Mengersen, K., Chao, C.Y.H., Wan, M.P., Li, Y., Xie, X., Katoshevski, D., and Corbett, S. (2011). Modality of human expired aerosol size distributions. J. Aerosol Sci. 42 (12):839-851. doi:10.1016/j.jaerosci.2011.07.009.

Lednicky, J.A., Shankar, S.N., Elbadry, M.A., Gibson, J.C., Alam, M., Stephenson, C.J., Eigurenfernandez, A., Morris, J.G., Mavian, C.N., Salemi, M., Clugston, J.R., and Wu, C. (2020). Collection of SARS-CoV-2 Virus from the Air of a Clinic within a University Student Health Care Center and Analyses of the Viral Genomic Sequence. Aerosol Air Qual. Res. 20:1167-1171. Lindsley, W.G., Blachere, F.M., Beezhold, D.H., Thewlis, R.E., Noorbakhsh, B., Othumpangat, S., Goldsmith, W.T., McMillen, C.M., Andrew, M.E., Burrell, C.N., and Noti, J.D. (2016). Viable influenza A virus in airborne particles expelled during coughs versus exhalations. Influenza Other Respi. Viruses 10 (5):404-413. doi:10.1111/irv.12390.

Liu, Yuan, Ning, Z., Chen, Y., Guo, M., Liu, Yingle, Gali, N.K., Sun, L., Duan, Y., Cai, J., Westerdahl, D., Liu, X., Ho, K., Kan, H., Fu, Q., and Lan, K. (2020). Aerodynamic analysis of SARS-CoV-2 in two Wuhan hospitals. Nature 582:557-560. doi:10.1038/s41586-020-2271-3.

Lu, J., Gu, J., Li, K., Xu, C., Su, W., Lai, Z., Zhou, D., Yu, C., Xu, B., and Yang, Z. (2020). COVID19 Outbreak Associated with Air Conditioning in Restaurant, Guangzhou, China, 2020. Emerg. Infect. Dis. 26 (7):20-23. doi:10.3201/eid2607.200764.

Miller, S.L., Nazaroff, W.W., Jimenez, J.L., Boerstra, A., Buonanno, G., Dancer, S.J., Kurnitski, J., 
Marr, L.C., Morawska, L., and Noakes, C. (2020). Transmission of SARS-CoV-2 by inhalation of respiratory aerosol in the Skagit Valley Chorale superspreading event. Indoor Air 1-10. doi:10.1111/ina.12751.

Morawska, L. and Cao, J. (2020). Airborne transmission of SARS-CoV-2: The world should face the reality. Environ. Int. 139 (April):105730. doi:10.1016/j.envint.2020.105730.

Morawska, L., Johnson, G.R., Ristovski, Z.D., Hargreaves, M., Mengersen, K., Corbett, S., Chao, C.Y.H., Li, Y., and Katoshevski, D. (2009). Size distribution and sites of origin of droplets expelled from the human respiratory tract during expiratory activities. J. Aerosol Sci. 40 (3):256269. doi:10.1016/j.jaerosci.2008.11.002.

Nicas, M., Nazaroff, W.W., and Hubbard, A. (2005). Toward understanding the risk of secondary airborne infection: Emission of respirable pathogens. J. Occup. Environ. Hyg. 2 (3):143-154. doi:10.1080/15459620590918466.

Pan, Y., Zhang, D., Yang, P., Poon, L.L.M., and Wang, Q. (2020). Viral load of SARS-CoV-2 in clinical samples. Lancet Infect. Dis. 20 (4):411-412. doi:10.1016/S1473-3099(20)30113-4.

Papineni, R. and Rosenthal, F. (1997). The size distribution of droplets in the exhaled breath of healthy human subjects. J. Aerosol Med. 10 (2):105-116. doi:10.1089/jam.1997.10.105.

Santarpia, J.L., Rivera, D.N., Herrera, V.L., Morwitzer, M.J., Creager, H.M., Santarpia, G.W., Crown, K.K., Major, D.M.B., Schnaubelt, E.R., Broadhurst, M.J., Lawler, J. V, Reid, S.P., and Lowe, J.J. (2020). Aerosol and surface contamination of SARS - CoV - 2 observed in quarantine and isolation care. Sci. Rep. 1-8. doi:10.1038/s41598-020-69286-3.

Schuit, M., Ratnesar-Shumate, S., Yolitz, J., Williams, G., Weaver, W., Green, B., Miller, D., Krause, M., Beck, K., Wood, S., Holland, B., Bohannon, J., Freeburger, D., Hooper, I., Biryukov, J., Altamura, L.A., Wahl, V., Hevey, M., and Dabisch, P. (2020). Airborne SARS-CoV-2 is Rapidly Inactivated by Simulated Sunlight. J. Infect. Dis. 222:564-571. doi:https://doi.org/10.1080/00498254.2020.1737890.

Smither, S.J., Eastaugh, L.S., Findlay, J.S., and Lever, M.S. (2020). Experimental Aerosol Survival of SARS-CoV-2 in Artificial Saliva and Tissue Culture Media at Medium and High Humidity. Emerg. Microbes Infect. 9:1415-1417. doi:10.1080/22221751.2020.1777906.

Tellier, R., Li, Y., Cowling, B.J., and Tang, J.W. (2019). Recognition of aerosol transmission of infectious agents: A commentary. BMC Infect. Dis. 19 (1):1-9. doi:10.1186/s12879-019-3707-y. van Doremalen, N., Bushmaker, T., Morris, D.H., Holbrook, M.G., Gamble, A., Williamson, B.N., Tamin, A., Harcourt, J.L., Thornburg, N.J., Gerber, S.., Lloyd-Smith, J.O., de Wit, E., and Munster, V.J. (2020). Aerosol and Surface Stability of SARS-CoV-2 as Compared with SARS- 
CoV-1. N. Engl. J. Med. 382:1564-1567.

Walker, J.S., Archer, J., Gregson, F.K.A., Michel, S.E.S., Bzdek, B.R., and Reid, J.P. (2020). Accurate Representations of the Microphysical Processes Occurring During the Transport of Exhaled Aerosols and Droplets. ACS Cent. Sci. doi:10.1021/acscentsci.0c01522.

Woelfel, R., Corman, V.M., Guggemos, W., Seilmaier, M., Zange, S., Mueller, M.A., Niemeyer, D., Vollmar, P., Rothe, C., Hoelscher, M., Bleicker, T., Bruenink, S., Schneider, J., Ehmann, R., Zwirglmaier, K., Drosten, C., and Wendtner, C. (2020). Virological assessment of hospitalized cases of coronavirus disease 2019. medRxiv 2020.03.05.20030502. doi:10.1101/2020.03.05.20030502.

World Health Organisation (2020). WHO Coronavirus Disease (COVID-19) Dashboard. Wu, A., Peng, Y., Huang, B., Ding, X., Wang, X., Niu, P., Meng, J., Zhu, Z., Zhang, Z., Wang, J., Sheng, J., Quan, L., Xia, Z., Tan, W., Cheng, G., and Jiang, T. (2020). Genome Composition and Divergence of the Novel Coronavirus (2019-nCoV) Originating in China. Cell Host Microbe 27 (3):325-328. doi:10.1016/j.chom.2020.02.001.

Wu, Z. and McGoogan, J.M. (2020). Characteristics of and Important Lessons from the Coronavirus Disease 2019 (COVID-19) Outbreak in China: Summary of a Report of 72314 Cases from the Chinese Center for Disease Control and Prevention. JAMA 323 (13):1239-1242. doi:10.1001/jama.2020.2648.

Xie, X., Li, Y., Chwang, A.T.Y., Ho, P.L., and Seto, W.H. (2007). How far droplets can move in indoor environments - revisiting the Wells evaporation-falling curve. Indoor Air 17:211-225. doi:10.1111/j.1600-0668.2006.00469,x.

Yan, J., Grantham, M., Pantelic, J., De Mesquita, P.J.B., Albert, B., Liu, F., Ehrman, S., and Milton, D.K. (2018). Infectious virus in exhaled breath of symptomatic seasonal influenza cases from a college community. Proc. Natl. Acad. Sci. U. S. A. 115 (5):1081-1086. doi:10.1073/pnas.1716561115.

Zayas, G., Chiang, M.C., Wong, E., MacDonald, F., Lange, C.F., Senthilselvan, A., and King, M. (2012). Cough aerosol in healthy participants: Fundamental knowledge to optimize droplet-spread infectious respiratory disease management. BMC Pulm. Med. 12 (March). doi:10.1186/1471-246612-11. 

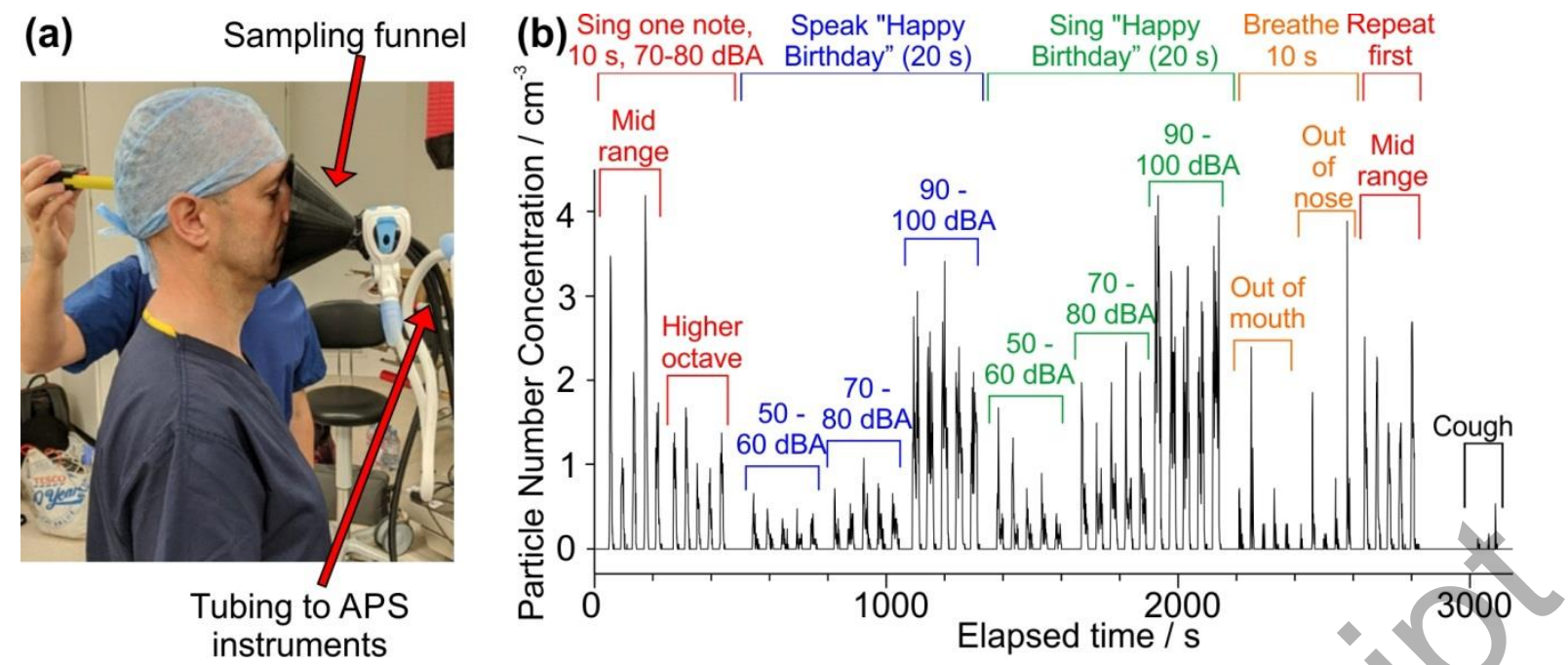

Figure 1: (a) An illustration of the sampling position of the performer, the sampling funnel and the aerosol instrumentation. (b) Continuous time series of data recorded from one participant completing 12 activities ( 5 repetitions of each). The zero-background is clearly apparent between measurements. 

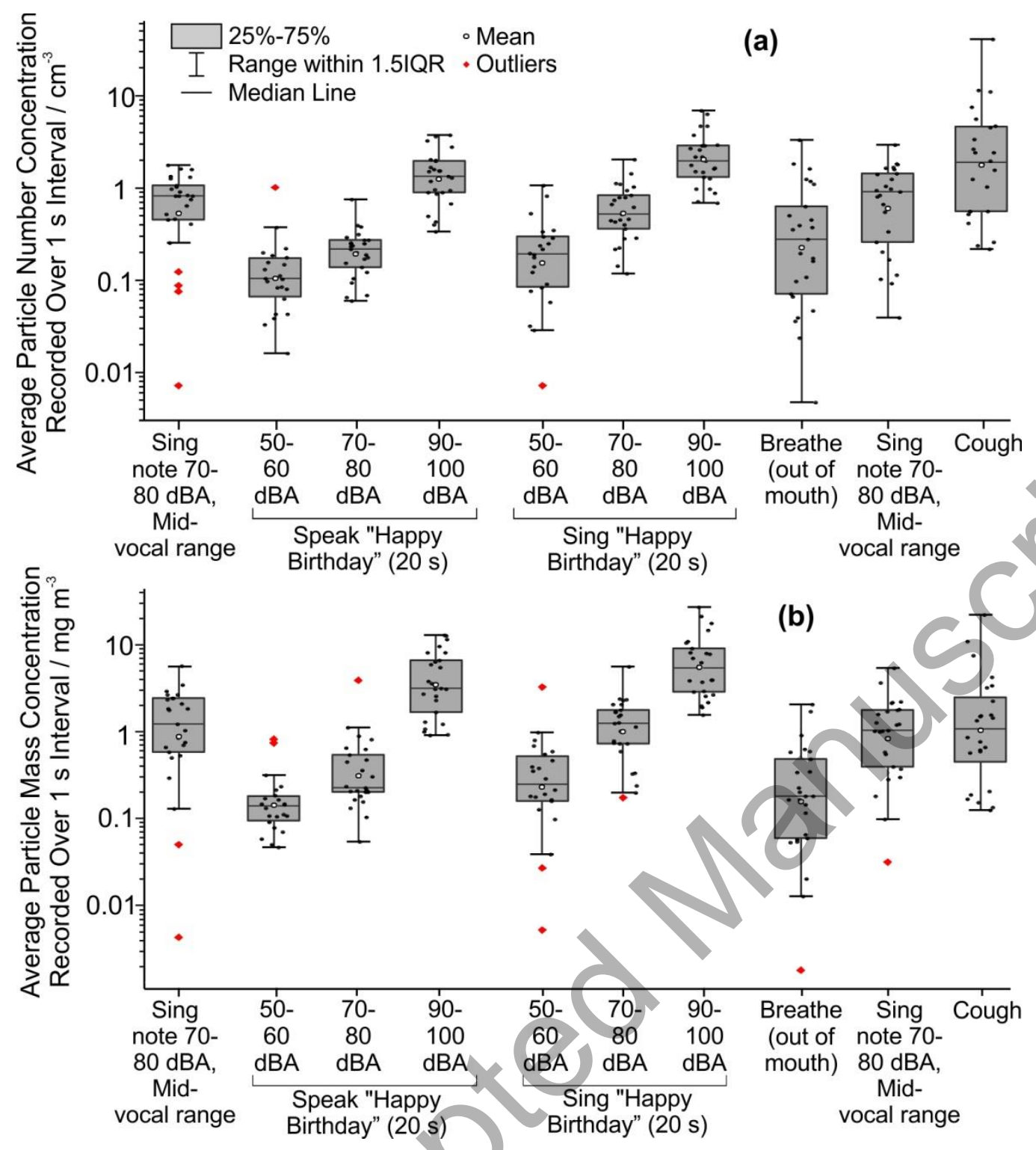

Figure 2: (a) Box and whisker plot showing particle number concentration and (b) mass concentrations for the same series of activities for all 25 participants. See Methods section ("Data and Statistical Analysis") for full description of analysis and reported values. 


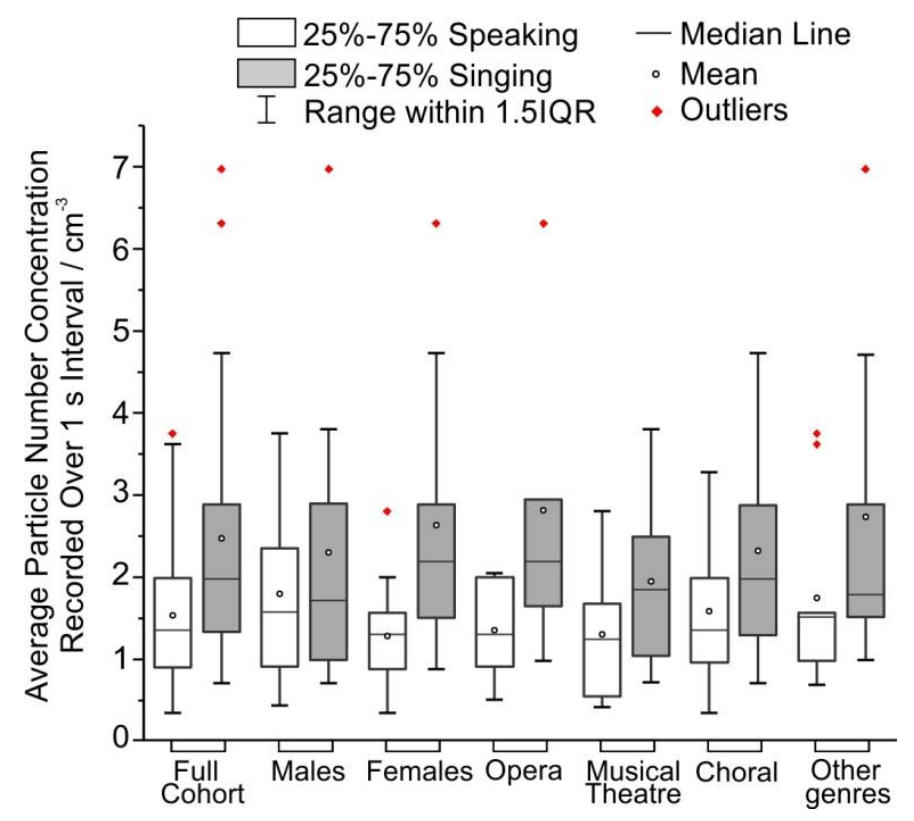

Figure 3: Comparison of average aerosol number concentrations (linear scale) from speaking and singing at 90-100 dBA by the full cohort, males (12), females (13), opera (5), musical theatre (6), choral (5) and other genres (9). 

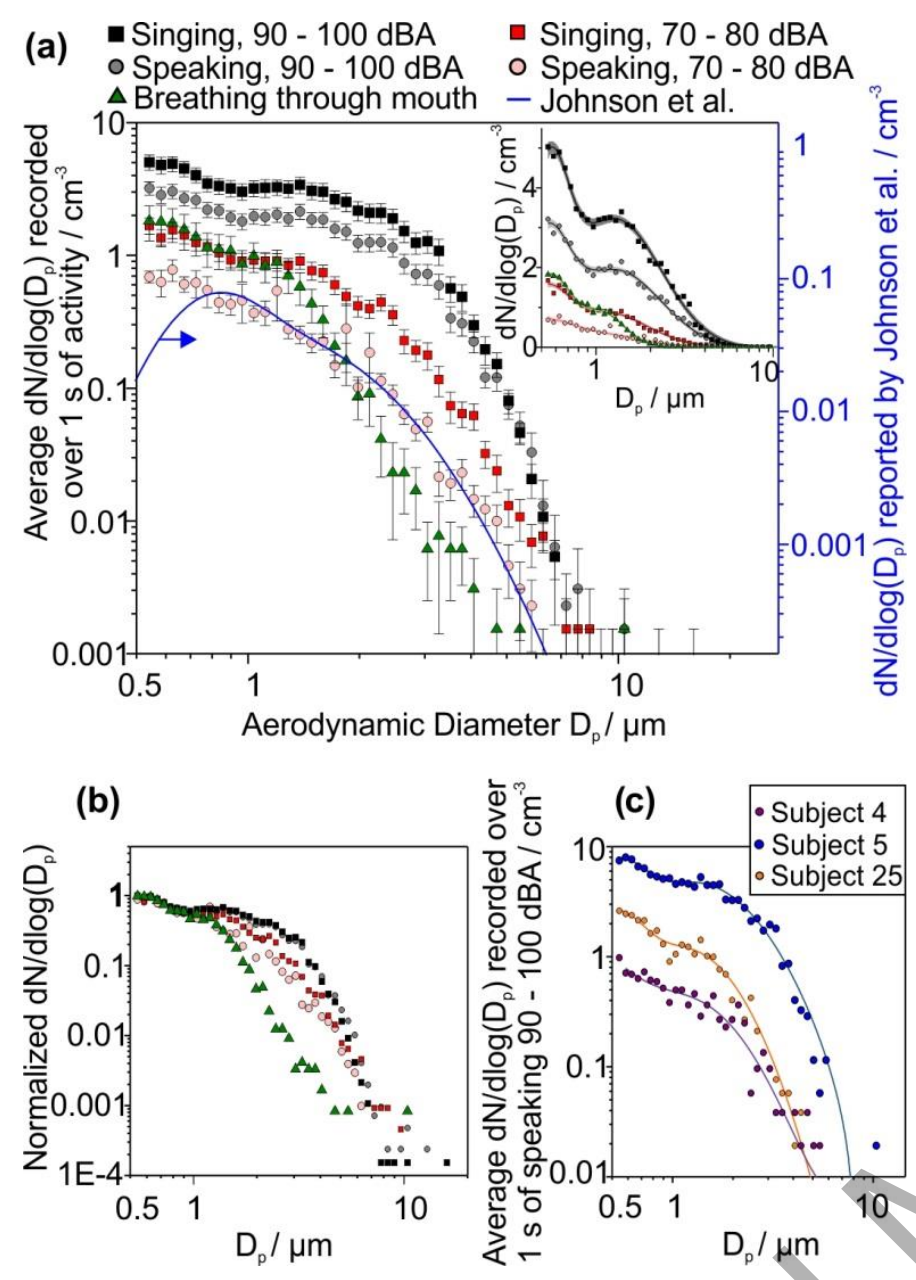

Figure 4: (a) Comparison of the size distributions from singing (squares) and speaking (circles) at different levels of loudness (70-80 dBA red; 90-100 dBA grey/black) with breathing (green triangles). The size distribution reported for speaking by Johnson et al.(Johnson et al. 2011) is shown by the blue line (right scale), data that should be most similar to the light red circles. The relative variations in concentrations represented by the two scales are equal. Error bars report standard error of the mean. The inset figure compares the fitted size distributions with the experimental data with a linear scale, as reported in Table S2. Shaded area about the fit line represents the 95\% confidence bound of the fit. (b) Comparison of the size distributions in (a) normalized with respect to the highest measured value of $\mathrm{d} N / \mathrm{d} \log \left(D_{p}\right)$ for each dataset. (c) Size distributions of the aerosol measured from three individual participants when speaking 90-100 dBA, i.e. data contributing to the mean size distributions represented by grey circles in (a). 
Table 1: The measured aerosol concentrations from different expiratory activities. Number concentration data correspond to the series of expiratory activities plotted in Figure $2 \mathrm{a}$ (in $\mathrm{cm}^{-3}$ ), and mass concentration correspond to the series of expiratory activities plotted in Figure $2 \mathrm{~b}$ (in $\mu \mathrm{g} \cdot \mathrm{m}^{-3}$ ). Provided are the statistical parameters visualized by the box and whisker plots. Note that these parameters were calculated on the logarithmically transformed data (see Materials and Methods). The number of participants for each activity is given by $n$.

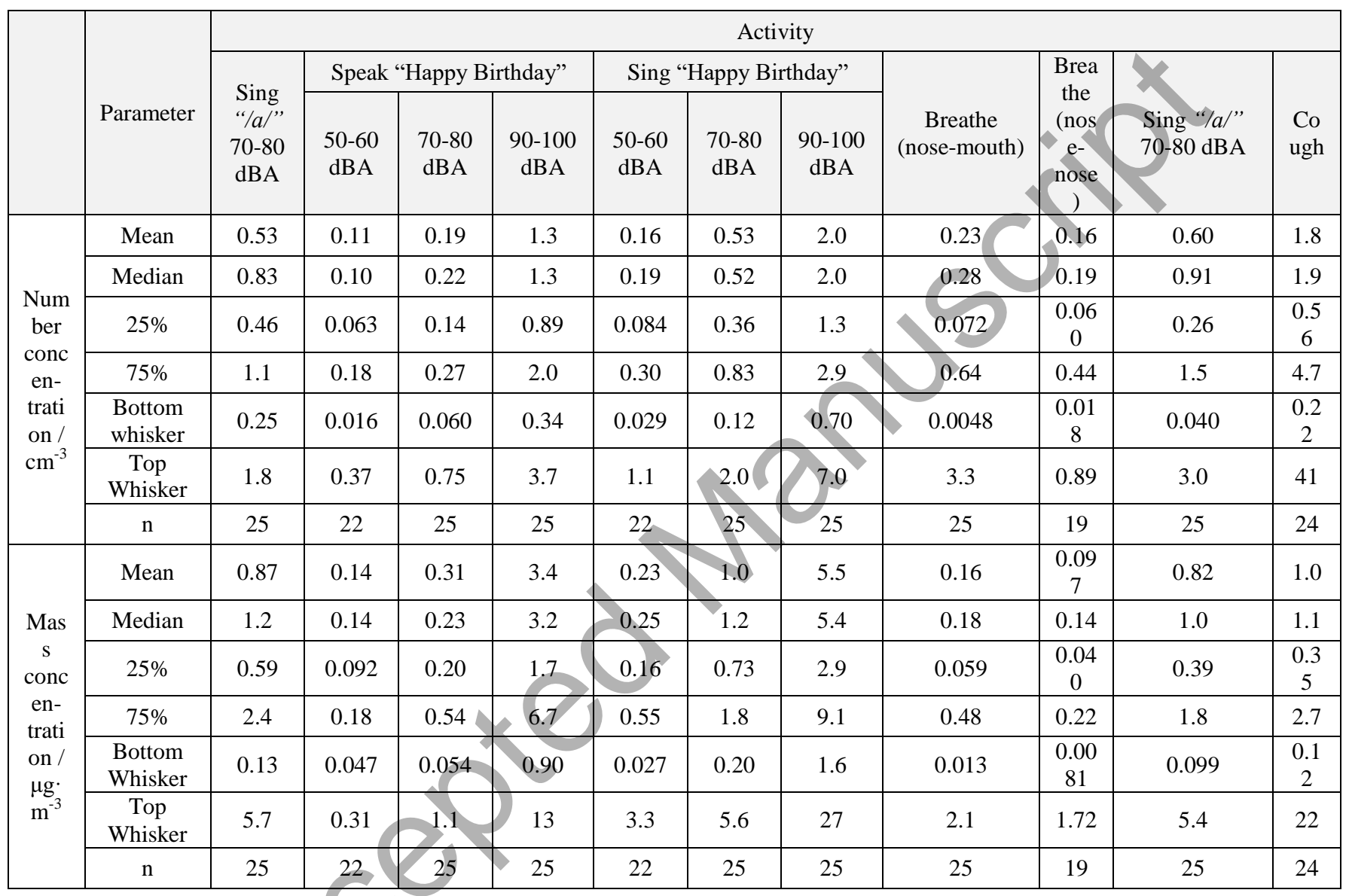

